\title{
An Emergency Alert Broadcast Based on the Convergence of 5G and ATSC 3.0
}

\author{
Yoon-Kwan Byun (D), Sekchin Chang * and Seong Jong Choi
}

Citation: Byun, Y.-K.; Chang, S.;

Choi, S.J. An Emergency Alert Broadcast Based on the Convergence of 5G and ATSC 3.0. Electronics 2021, 10, 758. https://doi.org/10.3390/ electronics10060758

Academic Editor: Martin Reisslein

Received: 24 January 2021

Accepted: 16 March 2021

Published: 23 March 2021

Publisher's Note: MDPI stays neutral with regard to jurisdictional claims in published maps and institutional affiliations.

Copyright: (c) 2021 by the authors. Licensee MDPI, Basel, Switzerland. This article is an open access article distributed under the terms and conditions of the Creative Commons Attribution (CC BY) license (https:/ / creativecommons.org/licenses/by/ $4.0 /)$.
Department of Electrical and Computer Engineering, University of Seoul, Seoul 02504, Korea; comkeen4@gmail.com (Y.-K.B.); chois@uos.ac.kr (S.J.C.)

* Correspondence: schang213@uos.ac.kr; Tel.: +82-2-6490-2342

\begin{abstract}
We propose a novel emergency alert broadcast mechanism for mobile phone users, which is based on the convergence of 5G and ATSC 3.0. Cellular networks including 5G adopt a broadcast technique for emergency alert. This technique just delivers a text-based message. Moreover, the message only includes a limited number of characters. Therefore, cellular networks cannot afford to provide abundant information in emergency cases. Broadcast networks such as ATSC 3.0 also offer an emergency alert broadcast service. This service can deliver a multimedia-based message in emergency cases. Therefore, the ATSC 3.0 supports more abundant information in the cases of emergency alert broadcasts. Especially, the ATSC 3.0 employs wake-up functionality and location information, which enables the delivery of emergency alerts to idle-state receivers in emergency areas. However, it is unlikely that the wake-up functionality and the location information are directly applicable to mobile phone users due to some practical issues. In order to improve the emergency alert broadcast service in mobile environments, we converge the 5G and the ATSC 3.0 networks, which effectively exploits the advantages of the networks. For the convergence network, we suggest a modified table, which associates the 5G message with the ATSC 3.0 message in the cases of emergency alerts. We also present a novel scenario for delivery of the emergency alert messages. Simulation results show that the convergence significantly enhances the receiver performance for emergency alert broadcast.
\end{abstract}

Keywords: emergency alert; broadcast; convergence; 5G; ATSC 3.0

\section{Introduction}

Recently, many cities rely on advanced technologies in order to achieve better life qualities. Especially, smart cities offer various automation applications such as autonomous vehicles and smart buildings [1]. The smart cities usually require robust emergency alert systems in order to sustain an acceptable safety level [2]. Therefore, much attention has been paid to advanced telecommunication technologies for rapid and reliable delivery of emergency alerts [3-5]. Among those technologies, cellular and broadcast networks are considered the most popular dissemination systems for emergency alerts [6-8].

The cellular networks employ cell broadcast service (CBS) for emergency alert delivery [9]. Since cellular networks can effectively select the base stations whose cells encompass emergency areas, the CBS is suitable for location-based emergency alert services [10]. Furthermore, the CBS is available even when mobile phones are in idle states, since cellular networks support a paging service for incoming calls [11]. Currently, the CBS protocols have been standardized for $2 \mathrm{G} / 3 \mathrm{G} / 4 \mathrm{G} / 5 \mathrm{G}$ cellular networks [12]. Therefore, any mobile phone users can receive the CBS messages in emergency cases if the users are located in the cells, which include emergency areas. However, the current CBS protocols just support text-based messages. Moreover, the CBS message allows a limited number of characters such as 360 characters in the case of wireless emergency alerts (WEA) CBS [13]. Therefore, the CBS message just includes limited information. In turn, it is impossible to deliver detail information such as evacuation guidelines. This may often lead to a failure in 
sustaining an acceptable safety level. Moreover, the current CBS protocols may be useless for illiterate persons or foreigners, since the protocols allow only text-based messages [14].

Broadcast networks also offer emergency alert services. Terrestrial digital multimedia broadcast (T-DMB) can deliver emergency alert messages even to mobile users [15-18]. However, T-DMB just supports text-based messages, which rely on automatic emergency alerting service (AEAS) [19]. Recently, advanced television systems committee (ATSC) 3.0 revealed about 20 standards for ultra-high definition (UHD) TV services and IP-based transmissions [20-23]. The ATSC 3.0 also specifies a standard for emergency alert broadcast, which is called advanced emergency information (AEA) [24]. One or more AEA messages include multimedia information as well as text data. Therefore, the ATSC 3.0 can deliver more abundant information such as guidelines in various emergency cases. In order to enable an idle-state receiver in the cases of emergency alerts, the ATSC 3.0 transmits wakeup information, which is similar to the paging service of cellular networks. However, most mobile phones including smart phones employ internal antennas. Therefore, the ATSC 3.0 receiver exhibits very poor receiver sensitivity in the internal antenna situation, since the ATSC 3.0 system operates at the carrier frequency of about $600 \mathrm{MHz}$ [25]. This may interfere with reliable usage of the wake-up information especially for mobile-phone users. Some complicated decoding algorithms were suggested for a reliable decoding of the wake-up information at low SNR levels [26,27]. However, the use of the algorithms may lead to excessive battery consumption. Therefore, the wake-up information is just available for TV receivers with external antennas. On the other hand, the paging service of the cellular networks is available even in the internal antenna case, since the cellular systems operate at much higher carrier frequencies such as 3.5 GHz. For location-based emergency alert services, location information can be embedded into the AEA messages. However, ATSC 3.0 receivers should employ a location-awareness functionality for the location-based services. Even though most smart phones employ GPS modules, the GPS devices do not guarantee high positioning accuracy. Therefore, some mobile users may fail in receiving the AEA messages due to the positioning error even in the cases that the users exist in emergency areas. Note that cellular networks inherently offer location-based emergency alert services, since the networks can easily select the appropriate base stations, which encompass emergency areas.

In order to improve the emergency alert broadcast service in mobile environments, we propose a novel emergency alert broadcast approach, which is based on the convergence of the 5G cellular network and the ATSC 3.0 network. Note that the 5G and the ATSC 3.0 are the latest protocols in cellular and broadcast networks, respectively. For the approach, we present a convergence architecture, which is suitable for the emergency alert broadcast. The convergence architecture employs thenon-3GPP interworking function (N3IWF) [28], which is an element of the 5G service based architecture (SBA) [29]. The N3IWF is standardized for interworking betweennon-3GPP network such as ATSC 3.0 network and 5G core network [28]. We also present a modified AEA table, which associates the AEA message with the corresponding CBS message. Based on the association, an alerting authority can reliably deliver an emergency message on the convergence network for emergency alert service. We also propose a novel scenario for the emergency alert service on the convergence network. Especially, the scenario specifies the role of the N3IWF for the broadcast of CBS and AEA messages on the convergence network. The scenario also specifies the relations among the CBS paging, the CBS message broadcast, and the AEA message broadcast. This allows a simultaneous transmission of CBS message and AEA message on the convergence network. Since the presented method effectively exploits the convergence, the scheme takes the advantages of the 5G and the ATSC 3.0 networks: multimedia information from the ATSC 3.0 network, and paging service and locationbased service from the $5 \mathrm{G}$ cellular network. Furthermore, the proposed method can lower delivery latency, since users can receive the CBS message and the AEA message from the 5G and the ATSC 3.0 networks, respectively. 
Some general architectures for combination of 5G and ATSC 3.0 networks have been suggested in [30] and [31]. However, the architectures [30,31] never reveal a specific framework for emergency alert broadcasts. Moreover, the architectures [30,31] never include a specific procedure for simultaneous delivery of 5G CBS message and AEA message. Therefore, the main contributions of this paper are as follows:

- We extended the general architectures into a 5G and ATSC 3.0 convergence network for emergency alert broadcast. The general architectures never reflect the interfaces among alerting authority, 5G CBS message generation, and AEA message application for the simultaneous delivery. We incorporated the interfaces into the convergence network. Furthermore, we added the functionality of CBS paging [8] to the convergence network, which enables mobile phones to receive AEA message as well as CBS message. Since the convergence network exhibits a unique architecture for emergency alert broadcast, the extension into the convergence network is a main contribution.

- We also proposed a novel framework for more reliable emergency alert broadcasts. The presented framework is utilized for a synchronization between CBS paging and AEA message in the 5G and ATSC 3.0 convergence network. The framework controls the delivery of AEA message, which guarantees that the AEA message is transmitted after the CBS paging is asserted. Since the framework exhibits a novel structure for the synchronization, the proposed framework is another contribution in this paper.

- In addition, we presented a novel procedure for simultaneous delivery of 5G CBS message and AEA message. Especially, the proposed procedure specifies the operations of the presented framework. The procedure guarantees that idle-state mobile phones can receive AEA message as well as CBS message using the CBS paging. Since the procedure exhibits a novel approach, which enables idle mobile phones to simultaneously receive AEA message and CBS message using the CBS paging, the presented procedure is also a contribution in this paper.

Simulation evaluation shows the validity of the proposed emergency alert broadcast approach. The simulation results exhibit that the proposed technique is much superior to the 5G CBS and the ATSC 3.0 AEA methods in terms of latency. This indicates that the presented approach allows more rapid notification in urgent situations. The results also reveal that the proposed method can effectively provide multimedia information as well as text-based information to users in emergency areas. The simulation results also show that various displays of emergency information are available on the receiver screen according to the decoding state. This indicates that the proposed approach is more reliable than the 5G CBS and the ATSC 3.0 AEA methods.

\section{Conventional Works}

As the conventional works for the emergency alert broadcast, we describe the 5G CBS and the AEA protocols, which are used in the $5 \mathrm{G}$ cellular and the ATSC 3.0 networks, respectively.

\subsection{G CBS Protocol}

5G cellular systems consist of user equipment (UE), radio access network (RAN), and core network $(\mathrm{CN})$. Among the system components, the UE indicates a cellular phone, and the RAN includes multiple base stations. The $\mathrm{CN}$ adopts the service based architecture (SBA) [29], which offers high flexibility and scalability. Therefore, 5G cellular systems can offer various services including enhanced mobile broadband (eMBB), ultra-reliable low latency communication (URLLC), and massive machine type communications (mMTC) [32].

In addition to those services, 5G cellular systems offer the CBS for emergency alert broadcast. Figure 1 illustrates the network architecture for the 5G CBS. The network architecture consists of alerting authority, cell broadcast entity (CBE), cell broadcast center function (CBCF), access and mobility function (AMF), RAN, and UE. In Figure 1, a central or a local government may act as the alerting authority. In Figure 1, CBCF and AMF belong to the $5 \mathrm{G}$ core network $(\mathrm{CN})$. When an emergency occurs, the alerting authority sends emergency message and location information (of emergency areas) to the CBE as shown 
in Figure 1. The CBE acts as a gateway between network operator and alerting authority. Therefore, the $\mathrm{CBE}$ receives the emergency message from the alerting authority, and relays the message to the $\mathrm{CBCF}$, which the network operator administrates. Then, the CBCF transforms the emergency message format into the CBS message format, which is specified by the 3GPP standard [12]. After the transformation, the CBCF sends the CBS message to the AMF. The AMF are interfaced to multiple RANs. Using the location information and tracking area information (TAI), the AMF selects the base stations for the emergency areas in the RANs. Then, the AMF sends the CBS message to the identified base stations.

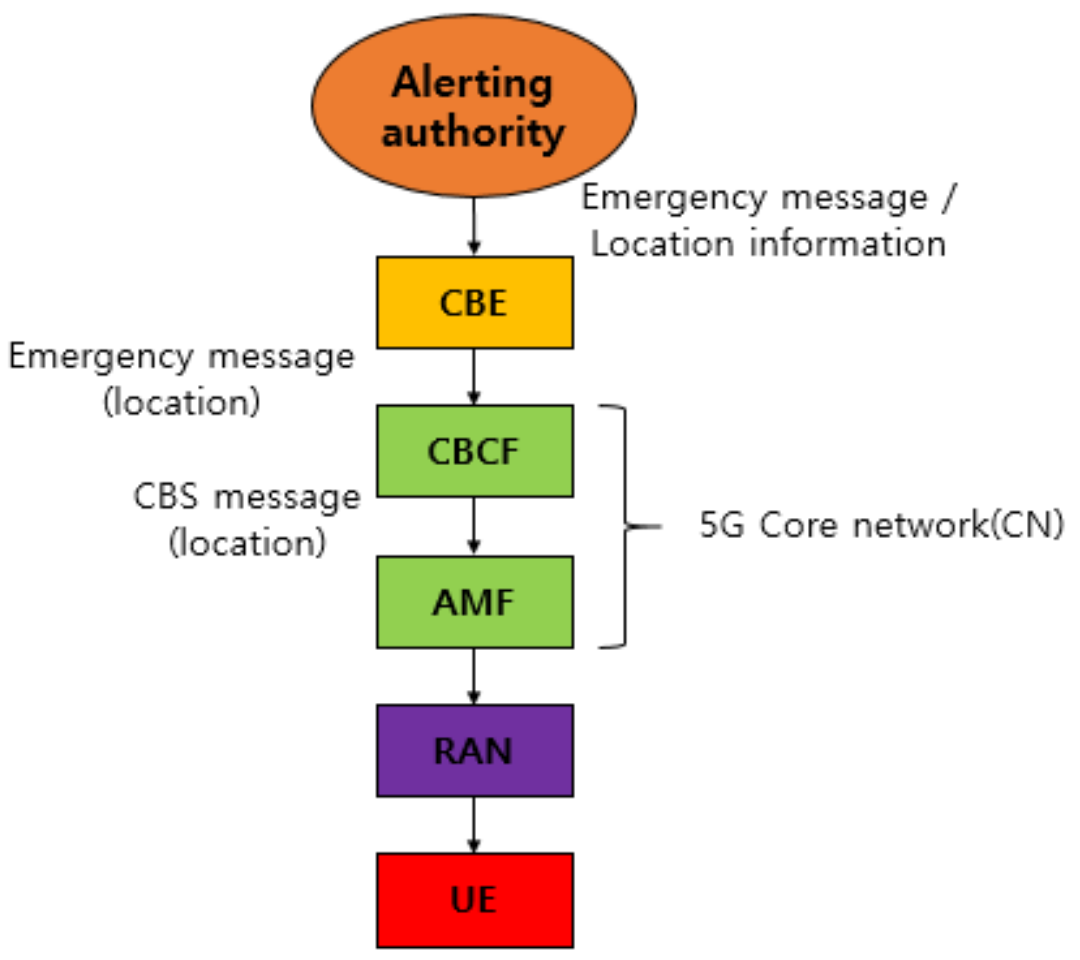

Figure 1. The network architecture for the 5G CBS.

In Figure 1, the CBS message is transferred from CBCF to base station (RAN) via wired channels. Figure 2 illustrates the network protocols for the CBS message transfer from CBCF to base station (RAN) [12].

As shown in Figure 2, the interface between CBCF and AMF relies on TCP/IP. The interface between AMF and base station (RAN) utilizes stream control transmission protocol (SCTP) and IP.

In Figure 2, the back-end of the base station (RAN) is connected to the AMF via a wired channel. However, the front-end of the base station is interfaced to the UEs (cellular phones) of Figure 1 via a wireless channel. Figure 3 illustrates the radio protocol for the wireless channel of the base station, which is used for the CBS message broadcast to all cellular phones in a cell. In Figure 3, the radio resource control (RRC) is responsible for the CBS paging and the CBS message broadcast [33]. If the base station receives a CBS message, the RRC of Figure 3 sends the paging message to all cellular users in a cell via the layer 2 and the layer 1. As indicated in Figure 3, the layer 2 includes packet data convergence protocol (PDCP), radio link control (RLC), and medium access control (MAC) sublayers. In Figure 3, the layer 1 indicates the physical layer (PHY). Among the interface channels between the sublayers, paging control channel $(\mathrm{PCCH})$, paging channel $(\mathrm{PCH})$, and physical downlink shared channel (PDSCH) are used for the paging broadcast in Figure 3. As stated earlier, the paging message enables the idle-state cellular phones to receive the CBS message. After the paging broadcast, the RRC sends the CBS message (SIB 8 ) to all cellular phones in a cell via layer 2 and layer 1 in Figure 3. For the CBS message 
broadcast, the CBS message is embedded into the system information block (SIB) 8 [33]. Among the interface channels between the sublayers, broadcast control channel $(\mathrm{BCCH})$, downlink shared channel (DL-SCH), and PDSCH are used for the CBS message (SIB 8) broadcast in Figure 3. The SIBs are usually utilized for the broadcast of control information (such as cell selection information) to all cellular phones in a cell. Therefore, the SIBs carry small-sized information. This indicates that the current 5G CBS protocol just allows a limited number of text characters such as 360 characters in the CBS message.

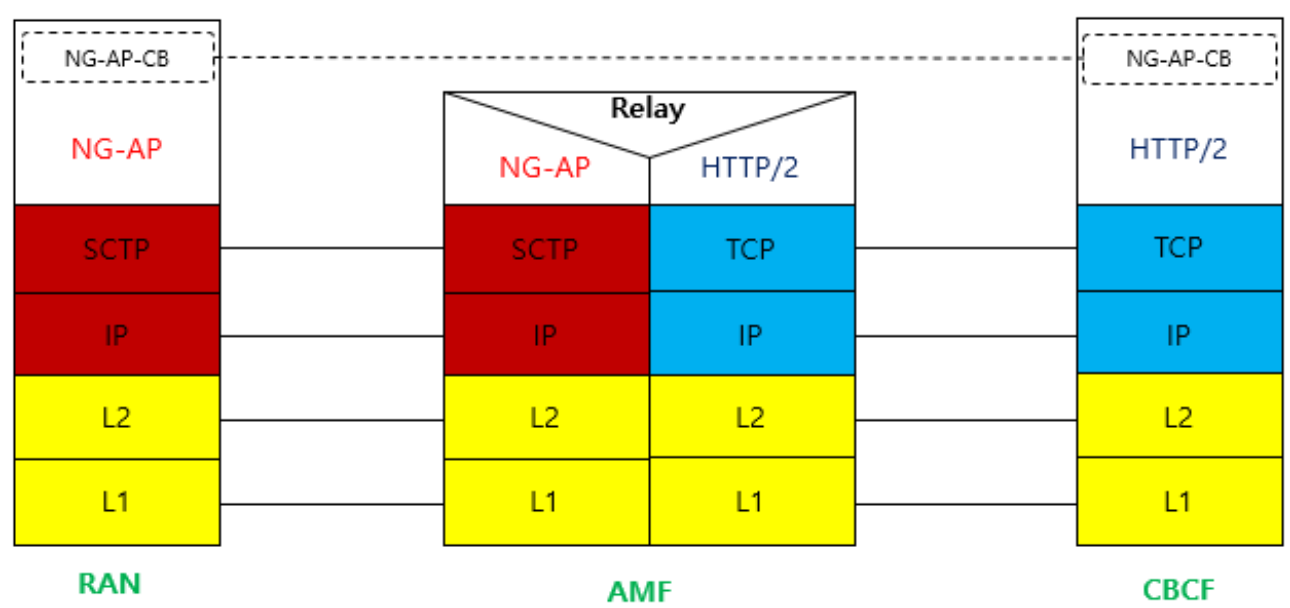

Figure 2. The network protocols for the CBS message transfer from CBCF to base station (RAN).

Layer 3

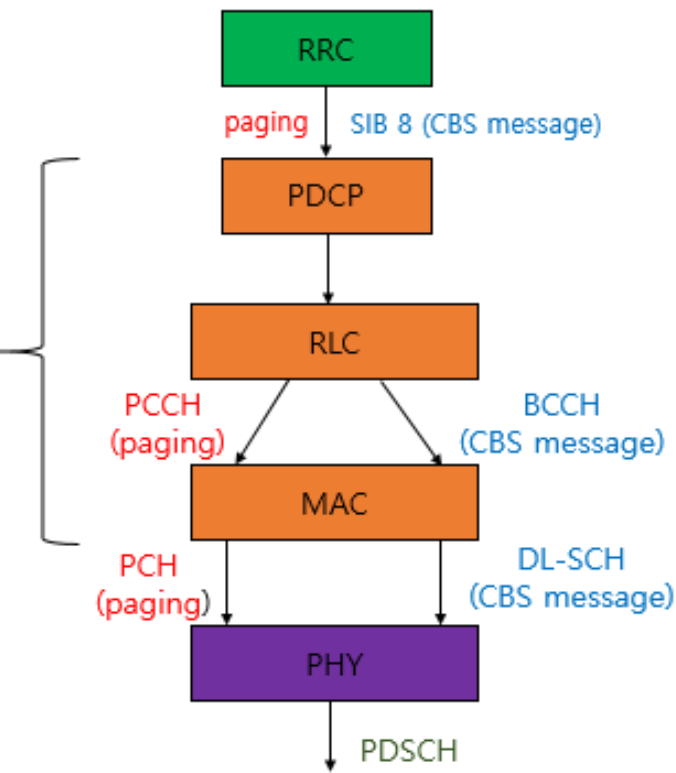

Figure 3. The radio protocol of the base station for 5G CBS.

\subsection{AEA Protocol}

ATSC 3.0 systems support the UHD TV services for better audio/video quality, and adopt the IP-based protocol for various broadcast and broadband services. Figure 4 illustrates the stack of the IP-based protocol for ATSC 3.0 [23]. 


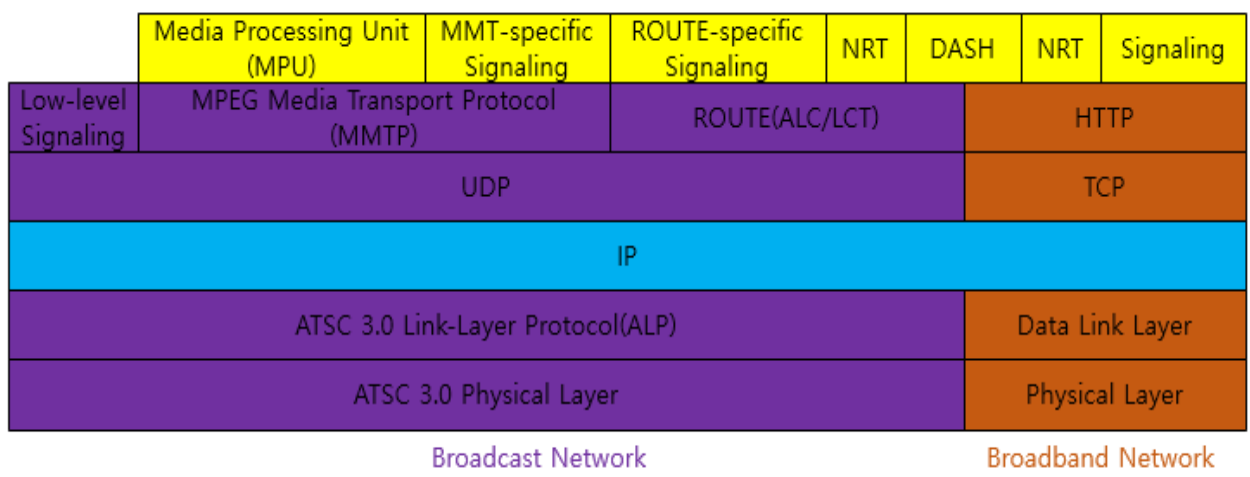

Figure 4. The stack of the IP-based protocol for ATSC 3.0.

In the ATSC 3.0, the real-time media components use the format of media processing unit (MPU) or the format of dynamic adaptive streaming over HTTP (DASH). As shown in Figure 4, the MPU components are transmitted on broadcast networks using MPEG media transport protocol (MMTP)/UDP/IP. The DASH components can be transmitted on broadcast networks or broadband networks using real-time object delivery over unidirectional transport (ROUTE)/UDP/IP or HTTP/TCP/IP, respectively. In the ATSC 3.0, the non-real time (NRT) components can be transmitted on broadcast networks or broadband networks using ROUTE/UDP/IP or HTTP/TCP/IP, respectively. The protocol stack of Figure 4 reveals that the real-time MPU components are transmitted on only broadcast networks, whereas the real-time DASH components and the NRT components can be transmitted on both broadcast and broadband networks. In Figure 4, the low level signaling (LLS) includes advanced emergency information table (AEAT), service list table (SLT), rate region table (RRT), system time, common alerting protocol (CAP), version information table (VIT), and content protection table (CPT). The LLS is transmitted on broadcast networks using UDP/IP. Since the AEAT consists of one or more AEA messages in the LLS, LLS/UDP/IP is used as the protocol for AEA message delivery in Figure 4.

Table 1 includes the description of the AEAT elements for one AEA message [24]. In Table 1, the emergency text and the multimedia information are associated with the elements of AEAText and Media, respectively. Furthermore, the AEAT includes the associated information for a live broadcast stream in the element of LiveMedia. Therefore, the ATSC 3.0 can provide a rich information (text, multimedia, and live broadcast stream) for emergency alert. This indicates that the ATSC 3.0 can provide much more abundant information for emergency alert than the 5G. In addition to the rich information, the ATSC 3.0 can deliver an emergency area information using the AEAT element of Header $<$ Location $>$. Using the area information, the receiver can discard the AEA message if the receiver user does not belong to the emergency area. However, the receiver is required to employ a location estimator such as GPS. A delicate location estimator usually causes high cost and high power consumption, which is not suitable for mobile terminals such as smart phones. A low-cost location estimator may give a poor estimation performance, which has a high potential to make a false discard of the AEA message. Note that the 5G CBS is very suitable for location-based emergency alert services, since the $5 \mathrm{G}$ AMF can select the appropriate base stations, which encompass emergency areas. 
Table 1. The description of the AEAT elements.

\begin{tabular}{|c|c|}
\hline Element & Description \\
\hline AEA & AEA message format \\
\hline$<$ aeaId $>$ & The identifier of AEA message \\
\hline <audience> & The intended distribution of the AEA message \\
\hline$<$ wakeup $>$ & $\begin{array}{c}\text { A true/false value for whether the wake-up field was activated for the emergency that the }<\text { aeaId }> \\
\text { message describes }\end{array}$ \\
\hline Header & The container for the basic alert envelope \\
\hline$<$ effective $>$ & The effective time of the AEA message \\
\hline$<$ expires> & The expires time of the AEA message \\
\hline$<$ EventCode $>$ & A code identifying the event type of the AEA message \\
\hline$<$ EventDesc $>$ & A short plain text description of the emergency event \\
\hline$<$ Location $>$ & The geographic code that identifies the affected area of the AEA message \\
\hline AEAText & The specific informative text of the AEA message \\
\hline LiveMedia & $\begin{array}{c}\text { The information of emergency-related real-time (live) audio/video service, which is delivered via } \\
\text { broadcast stream }\end{array}$ \\
\hline Media & The component parts of the multimedia resource \\
\hline$<$ lang $>$ & The code denoting the language of the respective element media \\
\hline$<$ mediaDesc $>$ & A short text description of the type and content of the media file \\
\hline$<$ mediaType $>$ & Text identifying the intended use of the associated media \\
\hline$<$ url $>$ & The identifier of the media file \\
\hline$<$ alternateUrl $>$ & Alternate URL of the media file when it is also available via non-broadcast delivery \\
\hline$<$ contentType $>$ & $\begin{array}{l}\text { The internet assigned numbers authority (IANA) media type of media content referenced by } \\
\qquad \text { Media<url> }\end{array}$ \\
\hline$<$ contentLength $>$ & The size (bytes) of media content referenced by Media $<$ url $>$ \\
\hline$<$ mediaAssoc $>$ & $\begin{array}{c}\text { The URI of another Media element with which this attribute is associated as described in } \\
<\text { <mediaType }>\end{array}$ \\
\hline
\end{tabular}

In Table 1, the element of AEA<wakeup> is associated with the wake-up service of ATSC 3.0. The wake-up service is used in order to activate an idle-state receiver for emergency alert service. For the wake-up service, the ATSC 3.0 transmits the wake-up field. The wake-up field is embedded in the bootstrap of the physical layer frame. Figure 5 illustrates the structure of the physical layer frame in ATSC 3.0 [22]. The bootstrap is used for wake-up information, synchronization, channel estimation, and preamble information. The wake-up field consists of two bits, each of which is located in bootstrap symbol 1 and bootstrap symbol 2, respectively.

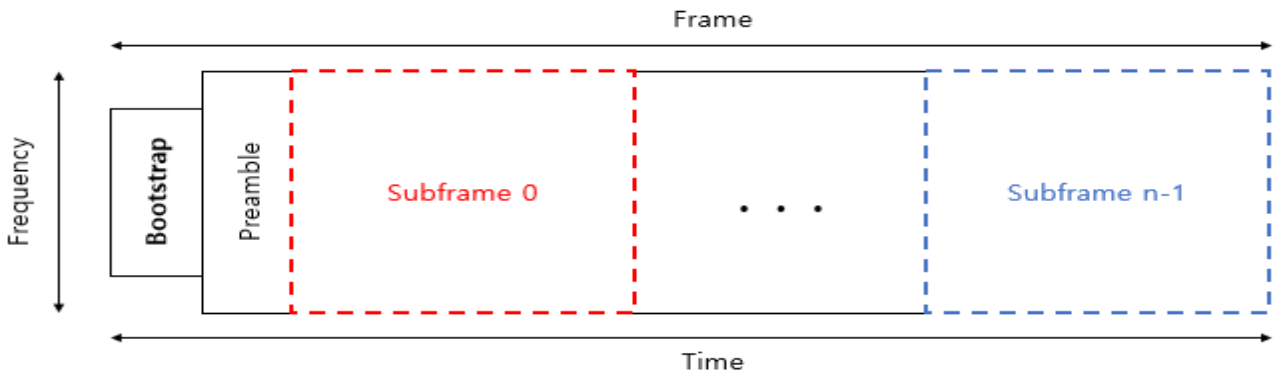

Figure 5. The structure of the physical layer frame in ATSC 3.0.

Table 2 exhibits the meaning of the AEA wake-up field [22]. Table 2 reveals that an idle-state receiver can be activated if the received wake-up filed is decoded into ' 01 ', ' $10^{\prime}$, or ' 11 '. After the activation, the receiver finds an AEA message corresponding to the wake-up field. The element of AEA<wakeup> is set to 'true' in Table 1 in the case that the AEA message corresponds to the wake-up field. However, it is unlikely that the wake-up service is available to smart phones. Note that most smart phones adopt internal antennas due to size limitations. The ATSC 3.0 receiver usually renders very poor receiver performance in the internal antenna case, since the ATSC 3.0 system operates at the carrier frequency of about $600 \mathrm{MHz}$ [25]. This prevents a reliable decoding of the wake-up field in the internal 
antenna situation. Some complicated decoding algorithms were suggested for a reliable decoding of the wake-up field at low SNR levels [26,27]. However, the adoption of the algorithms may lead to an excessive consumption of smart-phone battery. Therefore, the wake-up service is more suitable for fixed TV stations than smart phones. As stated in Section 2.1, the 5G CBS protocol adopts the paging service, which is similar to the wake-up service of ATSC 3.0. The paging service is available even in the internal antenna case, since $5 \mathrm{G}$ cellular systems operate at the carrier frequency of about $3.5 \mathrm{GHz}$.

Table 2. The meaning of the AEA wake-up field.

\begin{tabular}{cc}
\hline Value & Meaning \\
\hline 00 & No emergency to wake up devices is currently signaled \\
01 & Emergency to wake up devices-setting 1 \\
10 & Emergency to wake up devices-setting 2 \\
11 & Emergency to wake up devices-setting 3 \\
\hline
\end{tabular}

Therefore, it is required to converge the $5 \mathrm{G}$ and the ATSC 3.0 networks for better emergency alert service in mobile environments, which effectively takes the advantages of the 5G and the ATSC 3.0 systems. The convergence offers more reliable emergency alert service as well as rich alert information, which will be verified in Section 4.

\section{The Novel Emergency Alert Broadcast Approach Based on the Convergence}

\subsection{The Convergence Architecture and the Scenario}

Figure 6 illustrates the proposed overall architecture of the 5G and ATSC 3.0 convergence network for emergency alert broadcast.

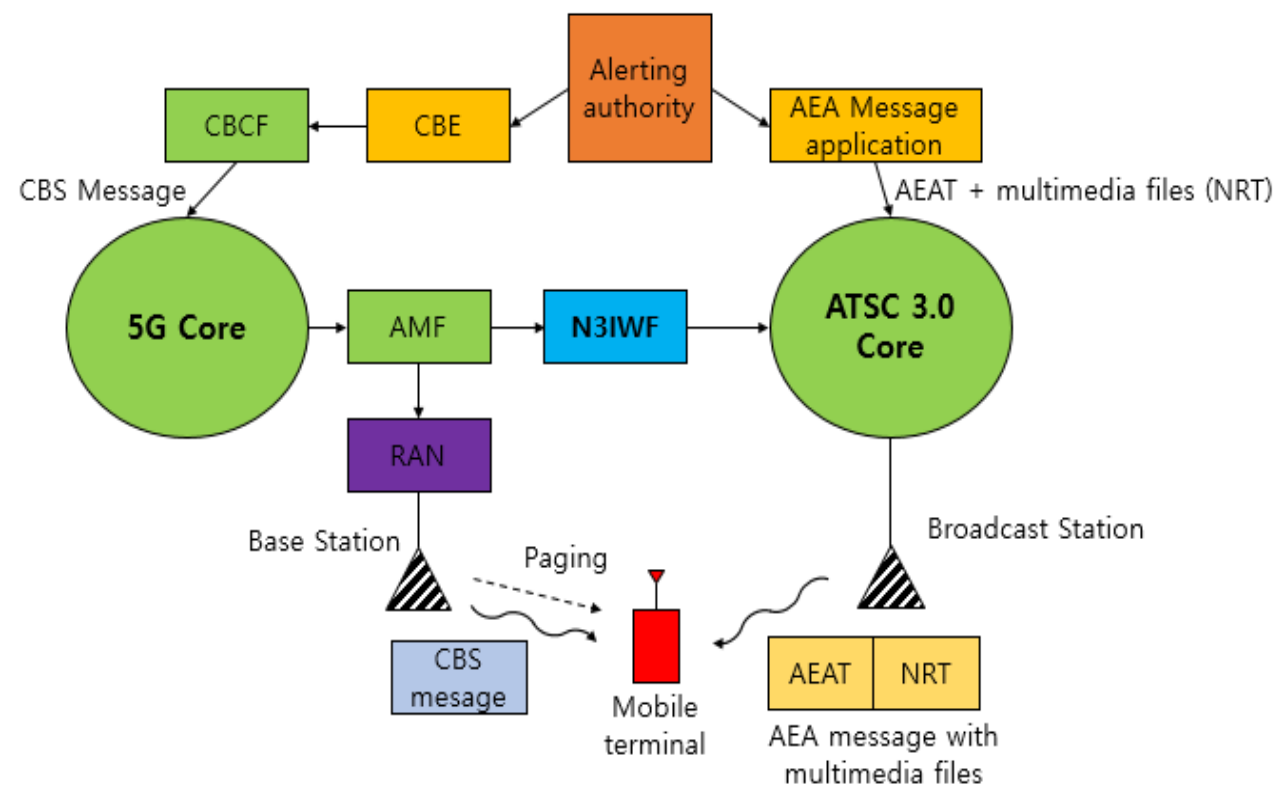

Figure 6. The overall architecture of the 5G and ATSC 3.0 convergence network for emergency alert broadcast.

The alerting authority simultaneously sends an emergency alert message to the 5G and the ATSC 3.0 systems. In the convergence architecture, the N3IWF is used for the convergence of the 5G and the ATSC 3.0 core networks. In Figure 6, the AEA message application produces the AEAT and the corresponding multimedia files, which belong to the NRT components. For an association with the CBS message, we propose a modified AEAT, which includes the additional CBS-related elements. Table 3 exhibits the additional elements. In Table 3, Header $<$ CBS flag $>$ indicates a Boolean value for whether the AEAT 
is associated with a CBS message for emergency alert broadcast on the convergence network of Figure 6. In Table 3, Header $<$ CBS ID> includes the identifier of the associated CBS message.

Table 3. The additional elements of the modified AEAT.

\begin{tabular}{|c|c|}
\hline Elements & Description \\
\hline Header & \\
\hline$<$ CBS flag $>$ & $\begin{array}{l}\text { A true/false value for whether the AEAT is } \\
\text { associated with the CBS message }\end{array}$ \\
\hline$<$ CBS ID $>$ & The identifier of the associated CBS message \\
\hline
\end{tabular}

In the convergence architecture of Figure 6, the scenario of the emergency alert broadcast includes the following steps:

1. When an emergency occurs, the alerting authority simultaneously sends an emergency alert message to the CBE/CBCF of the 5G and the AEA application of the ATSC 3.0.

2. The $\mathrm{CBCF}$ converts the emergency alert message to the $\mathrm{CBS}$ message according to the CBS protocol [12]. Then, the CBCF sends the CBS message to the AMF in the $5 \mathrm{G}$ core network. Based on the received emergency alert message, the AEA application generates the modified AEAT and the corresponding multimedia files (NRT components) according to the AEA protocol [24]. Then, the modified AEAT and the NRT components are transmitted to the ATSC 3.0 core network using the UDP/IP protocol.

3. The AMF simultaneously sends the CBS message to the N3IWF and the selected base stations of the RAN.

4. The N3IWF extracts the CBS ID (as a trigger information) from the CBS message, and sends it to the ATSC 3.0 core network.

5. The selected base stations broadcast the CBS paging signals to the mobile phones in the corresponding cells. As soon as the paging signals are received, the mobile phones become ready for the CBS message and the AEA message (which includes the modified AEAT and the NRT components) from the 5G base stations and the ATSC 3.0 broadcast stations, respectively.

6. In the ATSC 3.0 core network, the received trigger information causes the modified AEAT and the NRT components to be transmitted to the ATSC 3.0 broadcast stations.

7. The $5 \mathrm{G}$ base stations and the ATSC 3.0 stations broadcast the CBS message and the AEA message, respectively, to the mobile phones in the corresponding cells.

The steps 4,5 , and 6 reveal that the trigger information is used for a synchronization between the CBS paging signals and the AEA message. The trigger information causes the AEA message to be broadcast after the CBS paging signals are asserted.

Figure 7 illustrates the presented specific framework for the interface between AMF and ATSC 3.0 core network using the N3IWF. The ATSC 3.0 core network includes multiplexer, ATSC 3.0 gateway, and ATSC 3.0 modulator. In Figure 7, the novel procedure for simultaneous delivery of 5G CBS message and AEA message includes the following steps:

1. The AMF sends the CBS message to the N3IWF as well as the selected base stations. For the transmission of the CBS message, the AMF adopts the N2 protocol stack [34], which consists of $5 \mathrm{G}$ application protocol (5G-AP), SCTP, IP, layer 2 (L2), and layer 1 (L1). The 5G-AP includes the CBS message in Figure 7.

2. The N3IWF acts as a relay between the AMF and the ATSC 3.0 core network. For the connection to the AMF, the N3IWF adopts the N2 protocol stack. For the connection to the ATSC 3.0 core network, the N3IWF relies on the ATSC 3.0 protocol stack, which consists of ATSC 3.0 application protocol (ATSC3.0-AP), UDP, IP, ATSC 3.0 link layer protocol (ALP), and ATSC 3.0 PHY. The N3IWF extracts the CBS ID from the CBS message, and sends the CBS ID to the multiplexer in the ATSC 3.0 core network. The ATSC3.0-AP includes the extracted CBS ID in Figure 7. 
3. In the ATSC 3.0 core network, the multiplexer uses the CBS ID as the trigger information. If the received CBS ID is matched to the Header $<$ CBS ID $>$ of the modified AEAT, the multiplexer multiplexes the AEAT and the NRT components into the AEA message. Otherwise, the multiplexer suspends the multiplexing operation until the received trigger information is matched. Therefore, the CBS ID can be used as the trigger information for a synchronization between the CBS paging signal and the AEA message.

4. In the ATSC 3.0 core network, the ATSC 3.0 gateway transforms the AEA message into a frame unit on the physical layer. Then, the ATSC 3.0 gateway transmits the frame to the ATSC 3.0 modulator.

5. The ATSC 3.0 modulator converts the physical-layer frame to the waveform signal for broadcast.

6. The ATSC 3.0 broadcast station broadcasts the waveform signal for the AEA message.
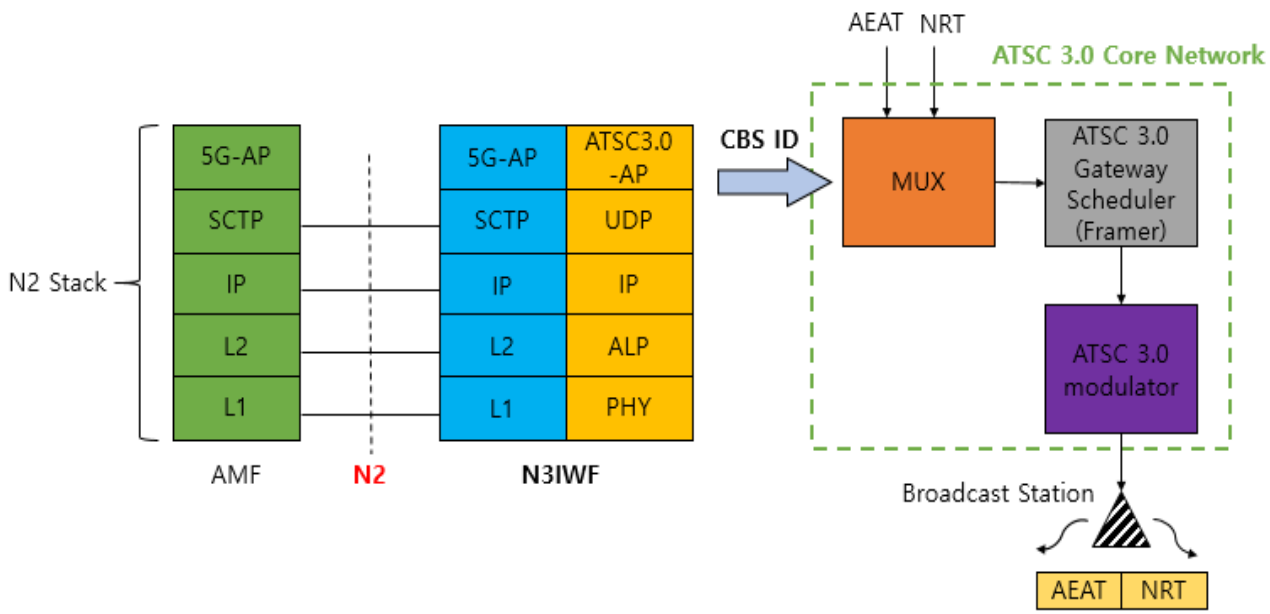

Figure 7. The specific framework for the interface between AMF and ATSC 3.0 core network using the N3IWF.

Some alternate architectures to the proposed approach were suggested in [30] and [31]. However, the architectures do not include some specific frameworks for emergency alert broadcasts as stated earlier.

\subsection{Latency Performace for the Proposed Emergency Alert Broadcast Approach}

In order to investigate the performance enhancement, we derive the latency performance for the proposed emergency alert broadcast approach. For the derivation, assume a random variable $\eta$, which denotes the number of total broadcasts for successful transmission of an emergency alert message to a mobile terminal. Thus, the expected value of $\eta$ denotes the latency performance, and is expressed as follows:

$$
E[\eta]=\sum_{k=0}^{\infty}(k+1) \operatorname{Pr}(K=k),
$$

where $K$ denotes the number of attempts required to successfully transmit the emergency alert message. $\operatorname{In}(1), \operatorname{Pr}(K=k)$ defines the probability that the first $k$ attempts will fail and the $(k+1)$ th attempt will succeed. Since $K$ follows a geometric distribution in (1), $\operatorname{Pr}(K=k)$ can be expressed as follows:

$$
\operatorname{Pr}(K=k)=p_{e}^{k}\left(1-p_{e}\right)
$$


where $p_{e}$ denotes the probability of an erroneous transmission of the emergency alert message to the mobile terminal in the case of the proposed convergence. After the substitution of (2) into (1), E[ $\eta]$ of (1) can also be expressed as follows:

$$
E[\eta]=\frac{1}{1-p_{e}} .
$$

Since the emergency alert broadcast method relies on the convergence network, $p_{e}$ of (3) can be expressed as follows:

$$
p_{e}=p_{e, 5 g} p_{e, a t s c 3}
$$

where $p_{e, 5 g}$ and $p_{e, a t s c 3}$ denote the probabilities of an erroneous transmission of the emergency alert message to the mobile terminal in the cases of the 5G and the ATSC 3.0, respectively. Therefore, the latency performance for the cases of the proposed convergence, the 5G, and the ATSC 3.0 can be given in (5), (6), and (7), respectively, as follows:

$$
\begin{gathered}
E[\eta]=\frac{1}{1-p_{e, 5 g} p_{e, a t s c 3}} . \\
E[\eta]=\frac{1}{1-p_{e, 5 g}} . \\
E[\eta]=\frac{1}{1-p_{e, a t s c 3}} .
\end{gathered}
$$

Since $p_{e, 5 g}<1$ and $p_{e, a t s c 3}<1, p_{e}$ of (4) is less than $p_{e, 5 g}$ and $p_{e, a t s c 3}$. Therefore, it is revealed that the latency performance for the proposed convergence is better than that for the $5 \mathrm{G}$ and the ATSC 3.0 in the comparison of (5), (6), and (7).

\section{Simulation Results}

Simulation results exhibit the effectiveness of the proposed emergency alert broadcast approach.

Table 4 summarizes the simulation parameters. The carrier frequencies of $5 \mathrm{G}$ and ATSC 3.0 networks are $3.5 \mathrm{GHz}$ and $600 \mathrm{MHz}$, respectively. As the fading channel models for 5G and ATSC 3.0, Jakarta [35] and TU-6 [36] channel models are adopted in this evaluation, respectively. In the channel models, each path follows Rayleigh distribution [37]. In this simulation, the cell radius for $5 \mathrm{G}$ is assumed to be $1 \mathrm{Km}$. In each cell, the number of receivers is set to 100 . The receivers are uniformly distributed in each cell. For the $5 \mathrm{G}$ base station and the ATSC broadcast station of Figure 6, it is assumed that the total transmit power is $1 \mathrm{~W}$ in this simulation.

Table 4. The simulation parameters.

\begin{tabular}{cc}
\hline Parameters & Value/Property \\
\hline Carrier frequency of 5G & $3.5 \mathrm{GHz}$ \\
Carrier frequency of ATSC 3.0 & $600 \mathrm{MHz}$ \\
Fading channel for 5G & Jakarta channel (Rayleigh) \\
Fading channel for ATSC 3.0 & TU-6 (Rayleigh) \\
Cell radius & $1 \mathrm{Km}$ \\
Number of receivers per cell & 100 \\
Distribution of the receivers & Uniform \\
Total transmit power & $1 \mathrm{~W}$ \\
\hline
\end{tabular}

Figure 8 exhibits the outage-state comparison of the proposed, the 5G, and the ATSC 3.0 emergency alert broadcasts after the first broadcast. 


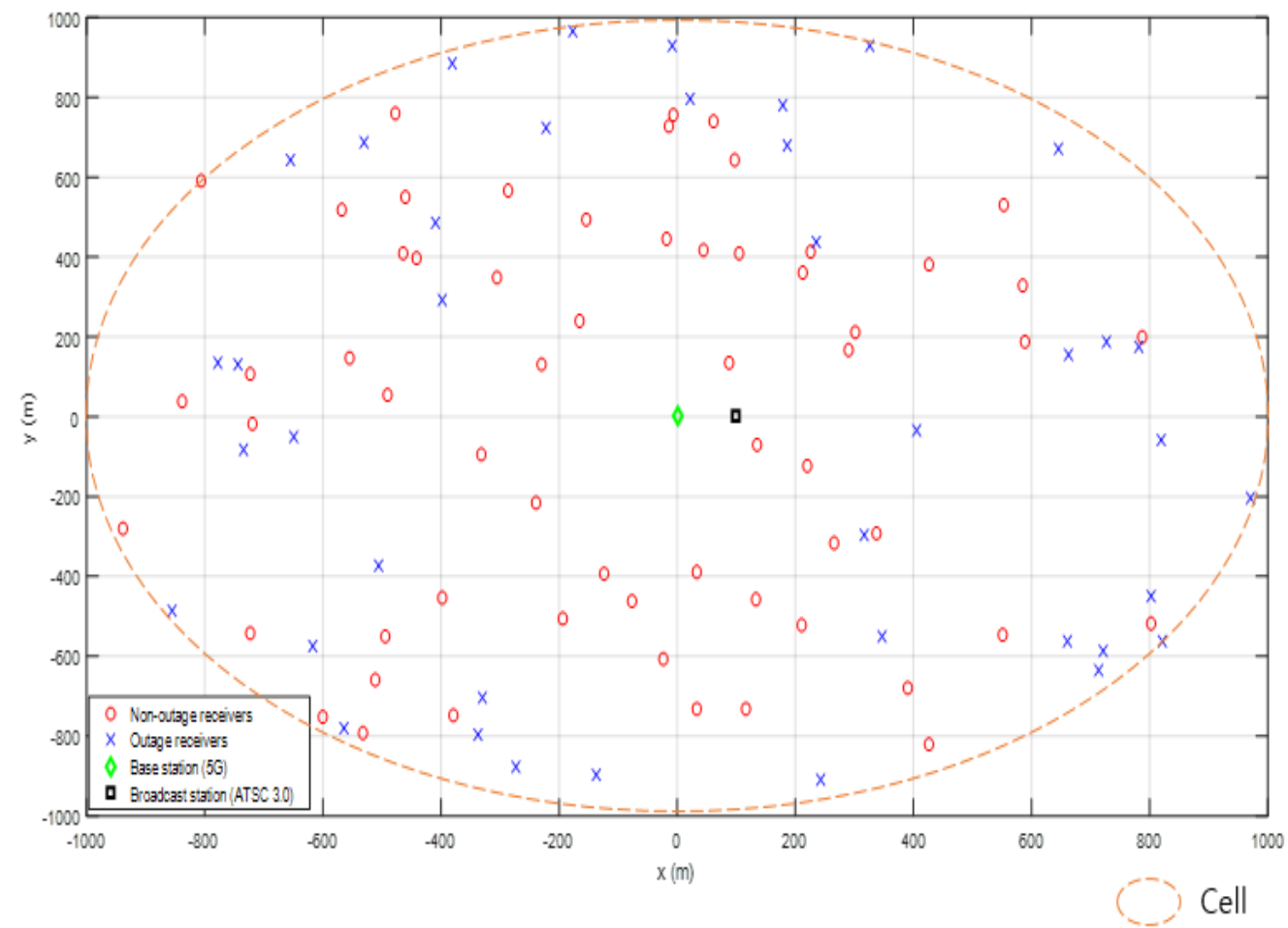

(a)

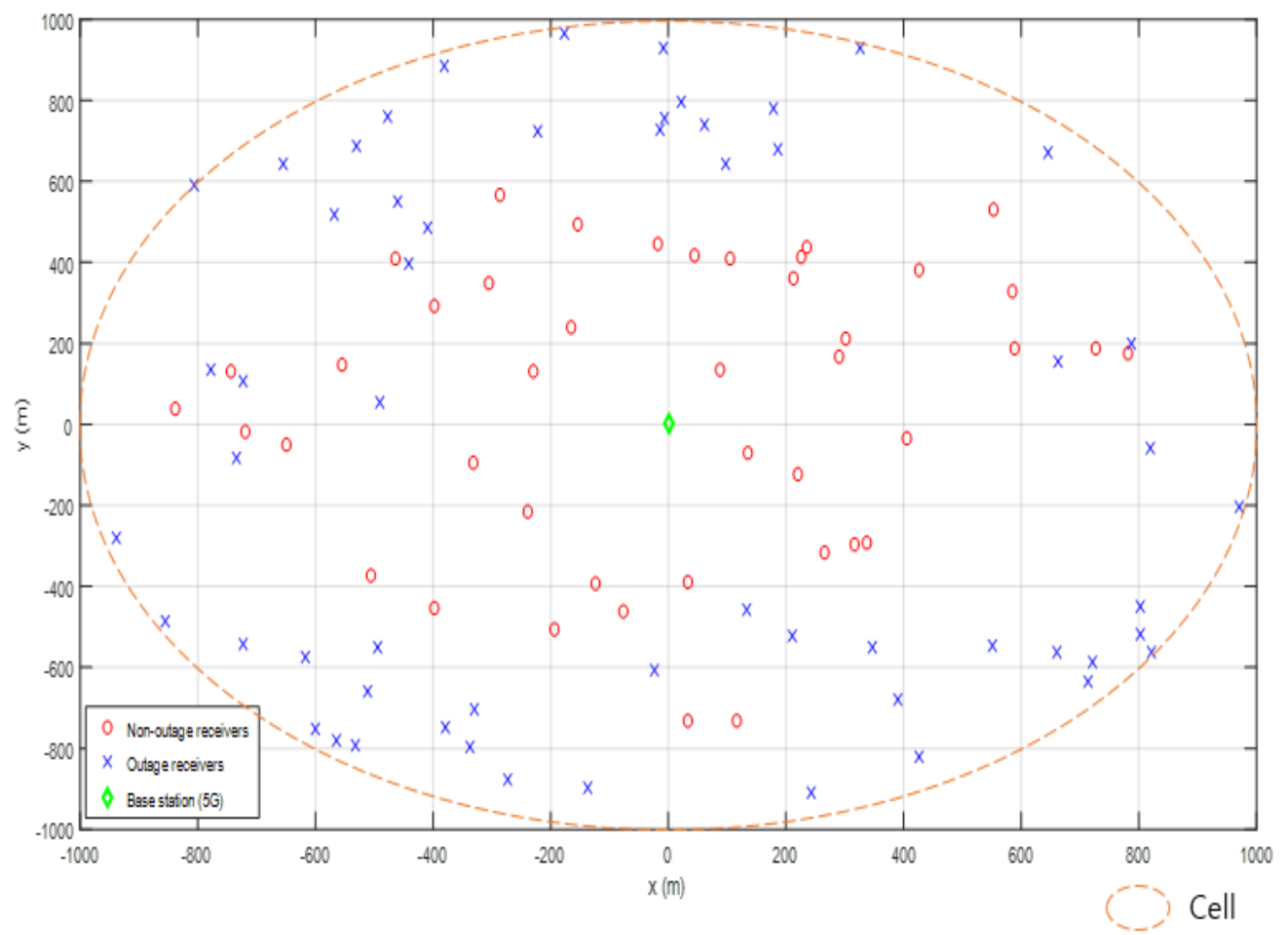

(b)

Figure 8. Cont. 


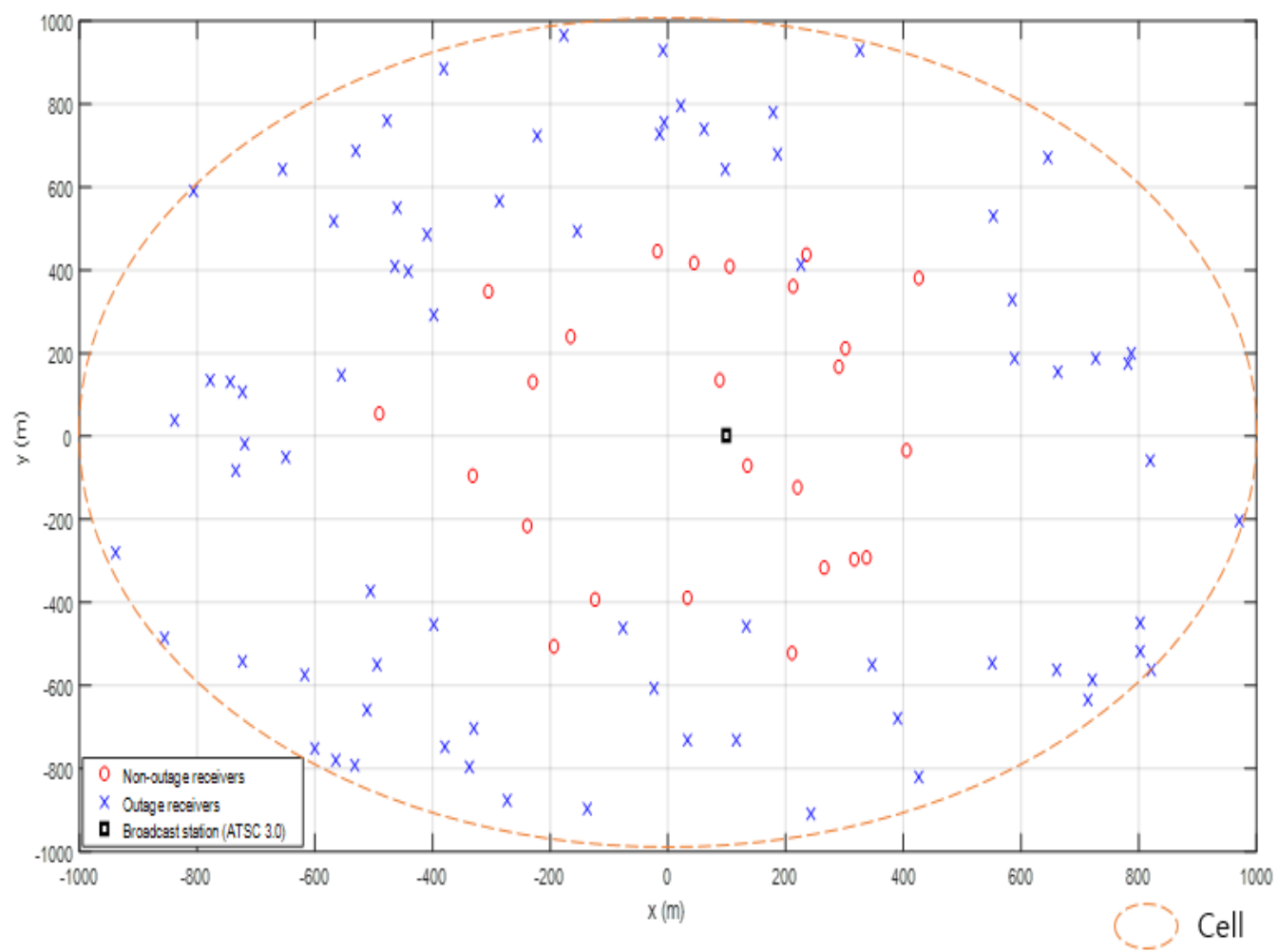

(c)

Figure 8. The outage-state comparison of the proposed, the 5G, and the ATSC 3.0 emergency alert broadcasts after the first broadcast. (a) shows the outage states for the proposed broadcast. This broadcast approach relies on the 5G and ATSC 3.0 convergence network of Figure 6. In the first broadcast, the 5G base station and the ATSC 3.0 broadcast station broadcast the CBS and the AEA messages, respectively. (a) reveals that the 60 receivers correctly decode the CBS message or the AEA message. Therefore, the success rate is $60 \%$ in the first broadcast case. (b) exhibits the outage states for the 5G CBS broadcast, which just relies on 5G cellular networks. The success rate is $43 \%$ in (b). (c) shows the outage states for the AEA message broadcast, which just relies on ATSC 3.0 networks. The success rate is $25 \%$ in (c). Since the users can receive the CBS message or the AEA message in the convergence network, the success rate of the proposed method is much higher than those of the 5G and the ATSC 3.0 cases.

Figure 9 exhibits the outage-state comparison of the proposed, the 5G, and the ATSC 3.0 emergency alert broadcasts after the second broadcast.

Figure 10 exhibits a comparison of the latency performance for the proposed, the 5G, and the ATSC 3.0 emergency alert broadcasts.

As shown in Figure 10, the latency of the proposed broadcast approach is much lower than those of the 5G CBS and the AEA broadcast methods over the entire range of $E_{b} / N_{0}$. Figure 10 reveals that proposed broadcast approach is much superior to the $5 \mathrm{G}$ CBS and the AEA broadcast methods in terms of latency. This indicates that the proposed convergence-based broadcast approach is more suitable for the emergency alert delivery, especially in the cases of urgent emergencies.

Figure 11 illustrates the comparison of terminal displays in the cases of success decoding of both 5G CBS and AEA messages, success decoding of only 5G CBS message, and success decoding of only AEA message for the proposed broadcast approach. 


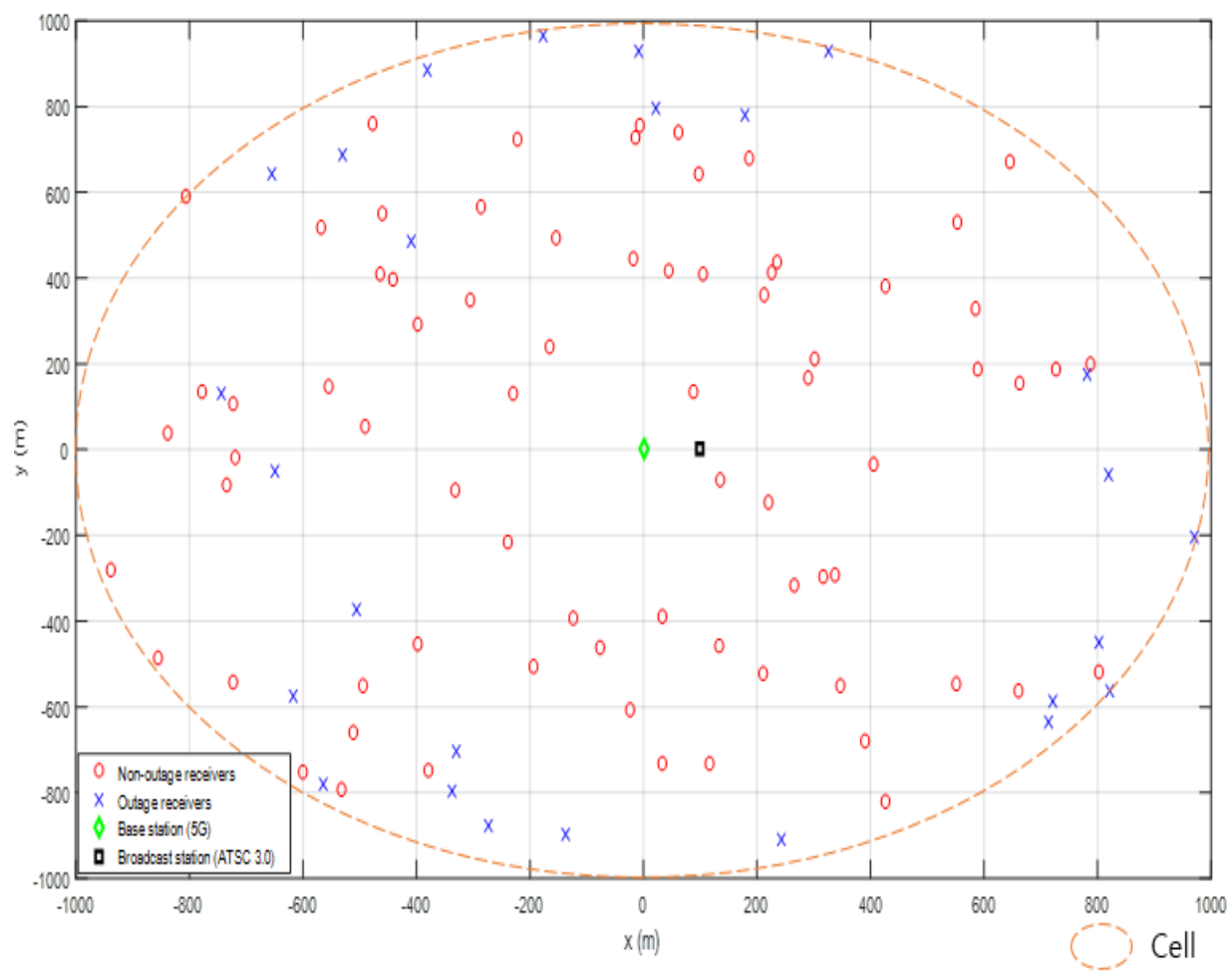

(a)

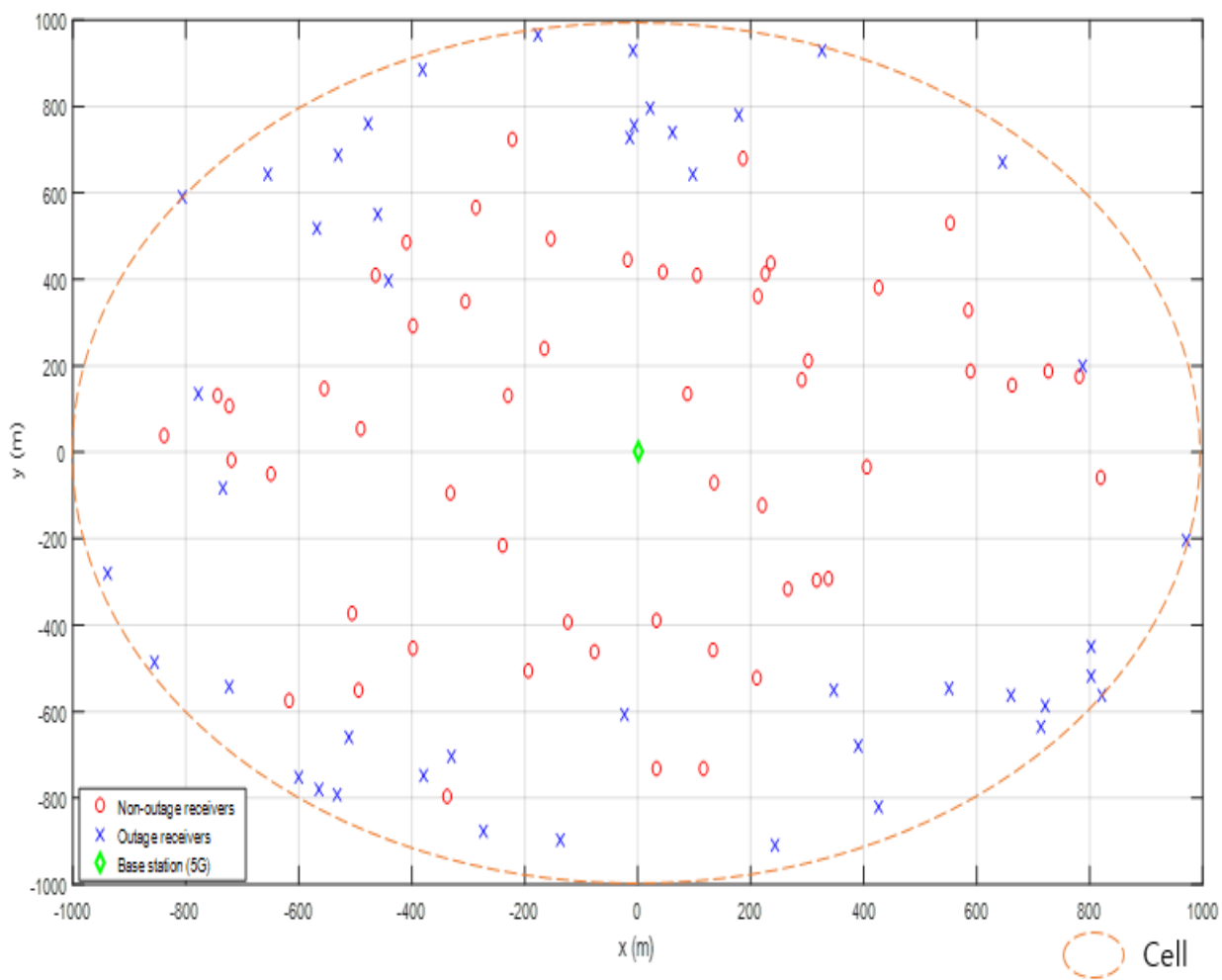

(b)

Figure 9. Cont. 


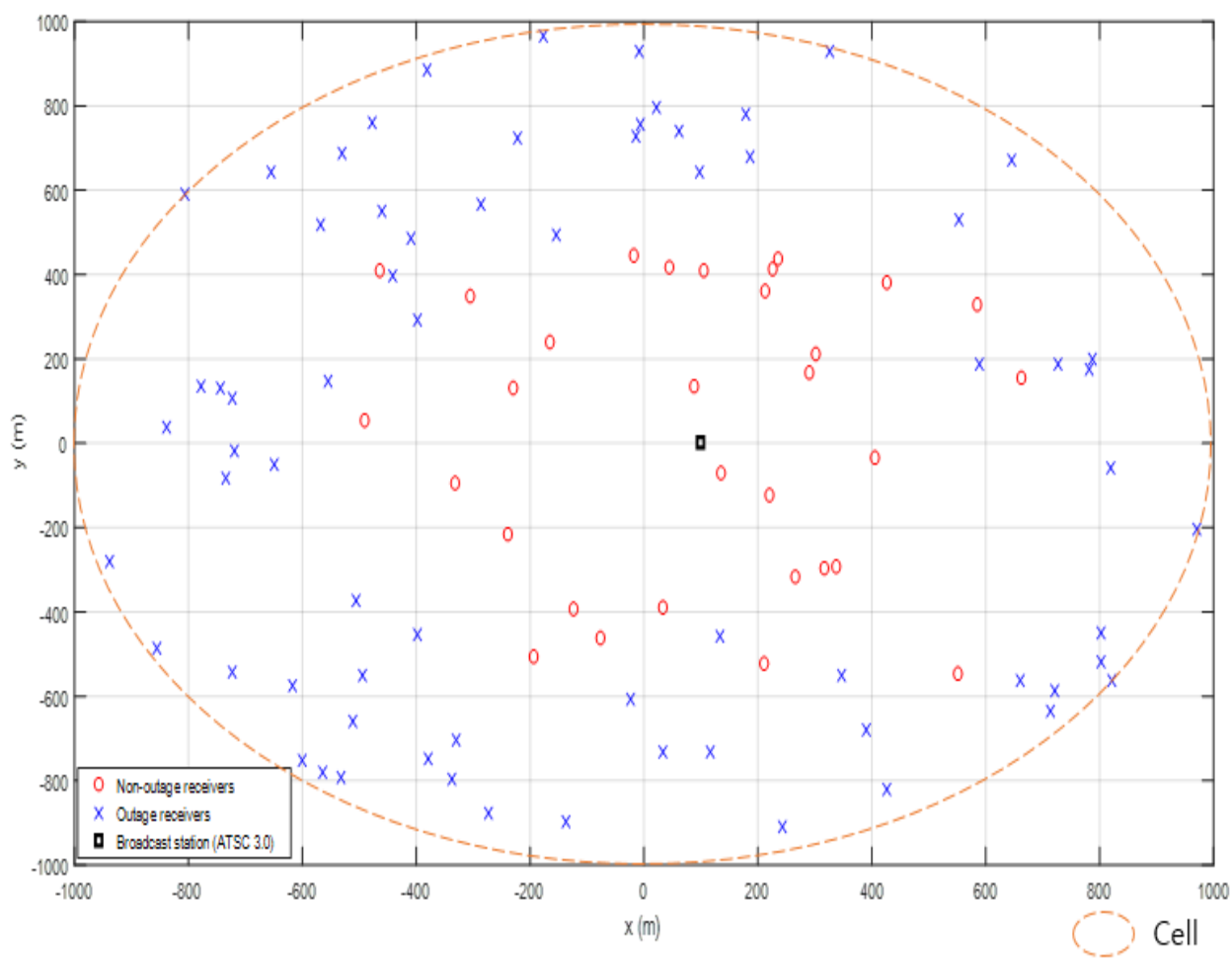

(c)

Figure 9. The outage-state comparison of the proposed, the 5G, and the ATSC 3.0 emergency alert broadcasts after the second broadcast. In (a), the success rate is $74 \%$. This indicates that most receivers can correctly decode the CBS message or the AEA message even within two broadcasts. This is due to the convergence network that simultaneously broadcasts the CBS message and the AEA message. (b) exhibits the outage states for the 5G CBS broadcast. Unlike the case of (a), there are still lots of outage receivers even after the second broadcast. In (b), the success rate is just $55 \%$. (c) shows the outage states for the AEA message broadcast. The success rate is $31 \%$ in (c). Therefore, more rebroadcasts are required in the cases of 5G and ATSC 3.0. Such latency may cause a serious effect, especially in urgent emergencies.

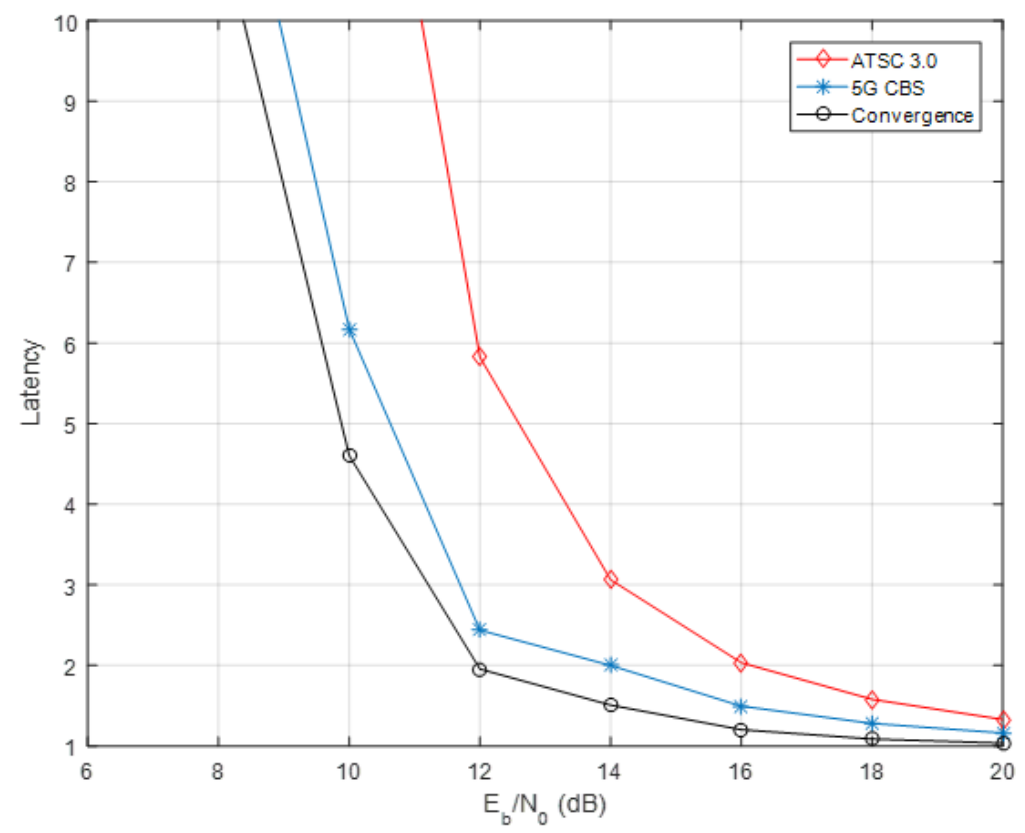

Figure 10. The comparison of the latency performance for the proposed, the 5G, and the ATSC 3.0 emergency alert broadcasts. 


\section{Conclusions}

We propose a novel emergency alert broadcast method in mobile environments, which considerably enhances the service qualities of emergency alert broadcast: lower delivery latency, more abundant emergency information, and higher accessibility of illiterate persons or foreigners. The presented broadcast approach is based on the convergence of the $5 \mathrm{G}$ cellular network and the ATSC 3.0 network. In this paper, we suggest a new architecture for the convergence. The convergence architecture exploits the N3IWF (an element of the 5G SBA) for interworking between ATSC 3.0 network and 5G core network. In order to associate the AEA message with the corresponding CBS message in the convergence architecture, we propose a modified AEAT, which includes CBS ID for the association of the AEA message with the corresponding CBS message. We also present a novel scenario for the emergency alert service on the convergence network. The scenario highlights the role of the N3IWF for the broadcast of CBS and AEA messages on the convergence network. For the simultaneous delivery of CBS message and AEA message on the convergence network, the scenario also specifies the relations among the CBS paging, the CBS message broadcast, and the AEA message broadcast. Since the convergence network takes the advantages of the 5G and the ATSC 3.0 systems, the proposed approach can afford to offer abundant emergency information to mobile phone users, including illiterate persons or foreigners. Furthermore, the presented method significantly lowers transmission latency, since the users can receive the CBS message and/or the AEA message from the 5G and the ATSC 3.0 networks, respectively.

Simulation results confirm the validity of the proposed emergency alert broadcast approach. The simulation evaluation exhibits that the presented method profoundly outperforms the 5G CBS and the ATSC 3.0 AEA methods in terms of latency. This reveals that the proposed approach is more suitable for urgent emergency cases. The results also indicate that the presented method can offer more abundant emergency information in addition to text-based information to mobile phone users including illiterate persons or foreigners. The simulation results also show that various emergency information (from abundant multimedia information to limited text information) is available according to the decoding state. This guarantees that the proposed approach is more reliable and flexible than the 5G CBS and the ATSC 3.0 AEA methods.
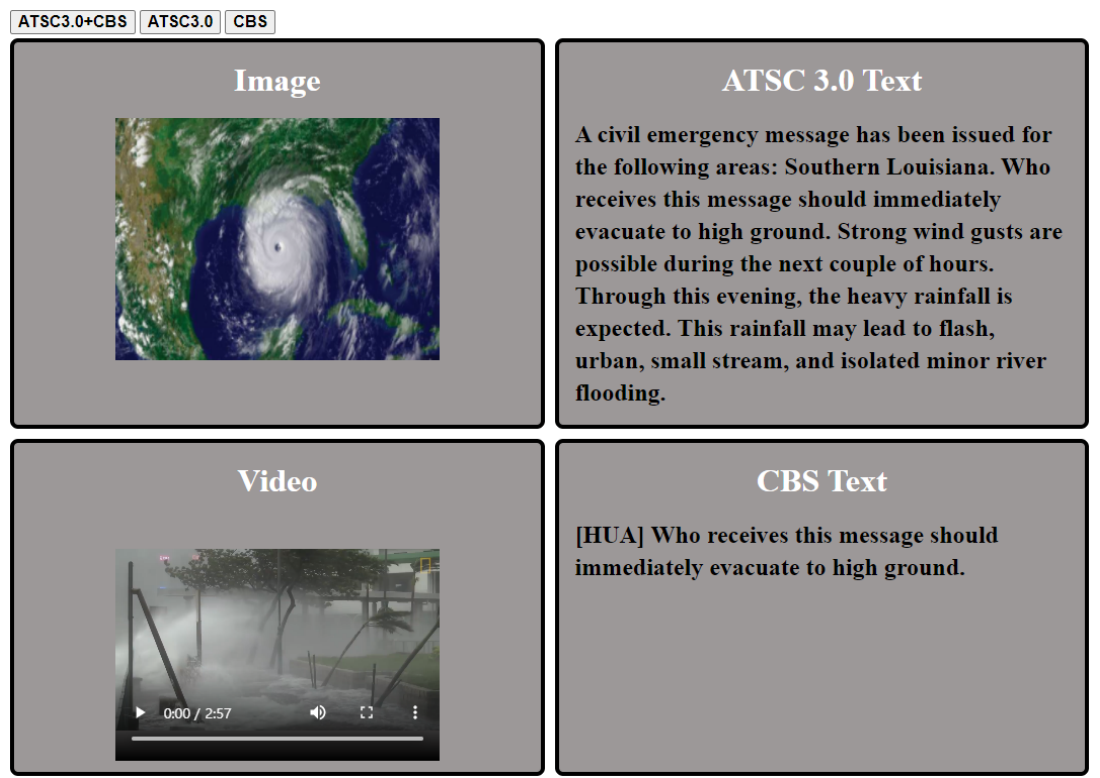

(a)

Figure 11. Cont. 

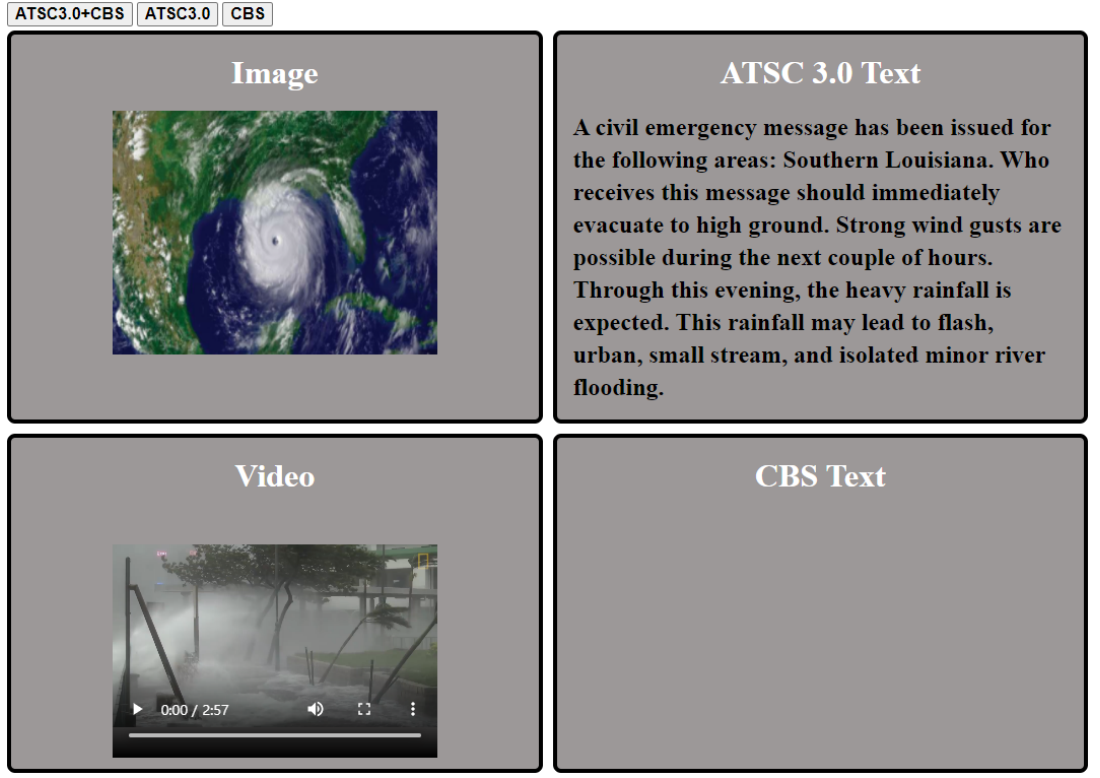

(b)
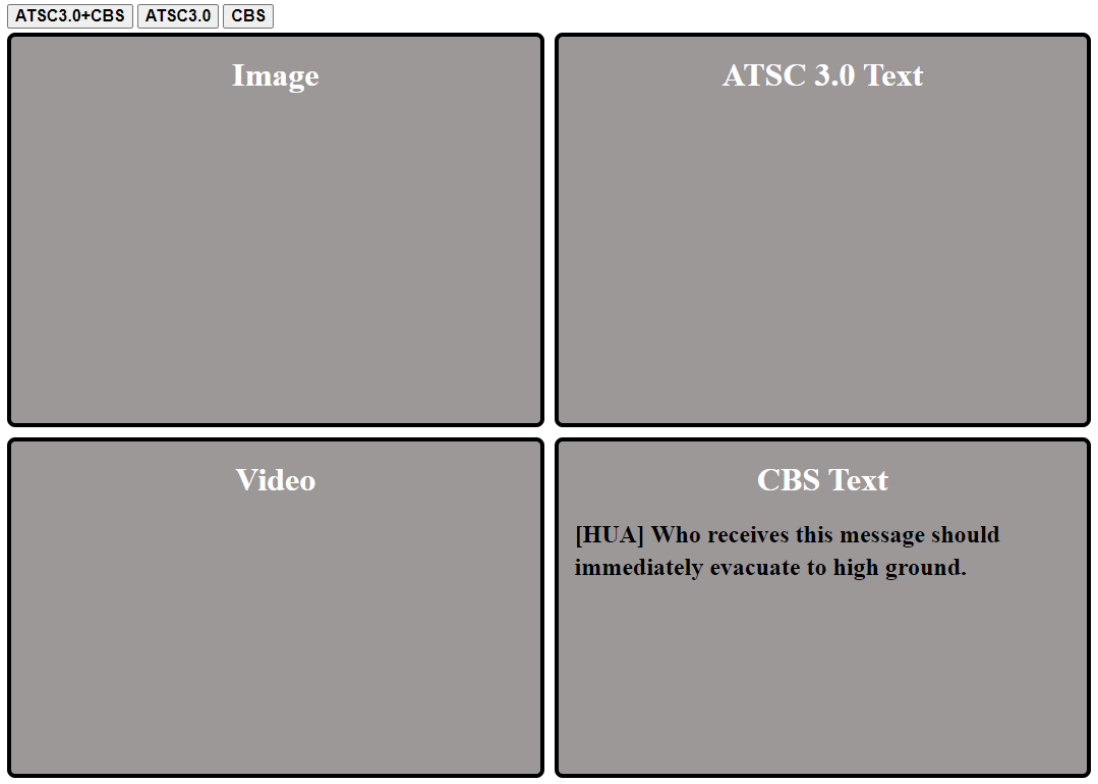

(c)

Figure 11. The comparison of terminal displays in the cases of success decoding of both 5G CBS and AEA messages, success decoding of only 5G CBS message, and success decoding of only AEA message for the proposed broadcast approach. We implemented the illustration of Figure 11 using HTML5. Therefore, the illustration of Figure 11 can be displayed on the screens of various platforms including smartphones. (a) shows the terminal display in the case of success decoding of both 5G CBS and AEA messages. As illustrated in (a), the terminal display includes the long text such as guideline, the image, the multimedia content of the AEA message as well as the short text of the CBS message. Therefore, even illiterate or foreign persons can easily recognize the emergency type such as earthquake or hurricane. (b) illustrates the case that the receiver failed in decoding the CBS message. As shown in (b), the sub-window for CBS text is blank. However, users can still achieve abundant emergency information from the AEA message. (c) illustrates the case that the receiver failed in decoding the AEA message. The sub-windows for AEA message are blank in (c). In this case, users can just achieve a limited emergency information from the CBS message. However, the information is still useful for most users. 
In order to further enhance the latency performance for the emergency alert broadcast approach, a cooperation scheme can be considered. However, note that the current 5G and ATSC 3.0 systems have distinct limitations (which include the relay capabilities of mobile phones, the scheduling issues for interference suppression, and network overhead) in incorporating such a cooperation technique into emergency alert broadcast scenario. In order to enable such a cooperation approach, we are going to focus on the developments of techniques for efficient relay capabilities, efficient device-to-device communications including interference-awareness scheduling, and simple relay-node management in further study.

Author Contributions: All authors contributed equally to this paper. All authors have read and agreed to the published version of the manuscript.

Funding: This research was supported by Disaster-Safety Platform Technology Development Program of the National Research Foundation of Korea (NRF) funded by the Ministry of Science and ICT (NRF-2018M3D7A1084906).

Conflicts of Interest: The authors declare no conflict of interest.

\section{References}

1. Kuru, K.; Khan, W. A framework for the synergistic integration of fully autonomous ground vehicles with smart city. IEEE Access 2020, 9, 923-948. [CrossRef]

2. Lee, Y.L.; Qin, D.; Wang, L.-C.; Sim, G.H. 6G massive radio access networks: Key applications, requirements and challenges. IEEE Open J. Veh. Technol. 2020, 2, 54-66. [CrossRef]

3. Javidroozi, V.; Shah, H.; Feldman, G. Urban computing and smart cities: Towards changing city processes by applying enterprise systems integration practices. IEEE Access 2019, 7, 108023-108034. [CrossRef]

4. Wang, F.; Pei, Z.; Dong, L.; Ma, J. Emergency resource allocation for multi-period post-disaster using multi-objective cellular genetic algorithm. IEEE Access 2020, 8, 82255-82265. [CrossRef]

5. Chang, D.; Cui, L.; Huang, Z. A cellular-automaton agent-hybrid model for emergency evacuation of people in public places. IEEE Access 2020, 8, 79541-79551. [CrossRef]

6. Elsawy, H.; Dai, W.; Alouini, M.-S.; Win, M.Z. Base station ordering for emergency call localization in ultra-dense cellular networks. IEEE Access 2017, 6, 301-315. [CrossRef]

7. Jeon, I.; Song, M.; Chang, S.; Choi, S.J.; Lee, Y.-T. A signaling emergency alert system multiplexed with T-DMB channel for emergency alert service. IEEE Trans. Consum. Electron. 2015, 61, 16-23. [CrossRef]

8. Song, M.; Jun, K.; Chang, S. An efficient multiplexing method of T-DMB and cell broadcast service in emergency alert systems. IEEE Trans. Consum. Electron. 2014, 60, 549-557. [CrossRef]

9. Chang, S. A synchronous cooperative communication for emergency alert broadcast based on cellular systems. J. Broadcast Eng. 2014, 19, 184-194. [CrossRef]

10. Zhu, Y.; Gao, X.; Willson, J.K.; Ma, C.; Jue, J.P.; Wu, W. Improving cell broadcasting scheme to support multi-lingual service in wireless networks. IEEE Commun. Lett. 2009, 13, 634-636. [CrossRef]

11. Sengupta, A.; Alvarino, A.R.; Catovic, A.; Casaccia, L. Cellular terrestrial broadcast-physical layer evolution from $3 \mathrm{GPP}$ release 9 to release 16. IEEE Trans. Broadcast. 2020, 66, 459-470. [CrossRef]

12. 3GPP TS 23.041 V16.2.0: Technical Realization of Cell Broadcast Service (CBS). Release 16. October 2019.

13. ATIS-0700023: Feasibility Study of LTE WEA Message Length. October 2015.

14. ATIS-0700026: Feasibility Study for WEA Supplemental Text. December 2015.

15. Kim, M.; Chang, S. A novel receiver for reliable IoT communications based on Zigbee under frequency-selective indoor environments. IEICE Trans. Fundam. 2017, e100-a, 361-365. [CrossRef]

16. Kim, M.; Chang, S. A consumer transceiver for long-range IoT communications in emergency environments. IEEE Trans. Consum. Electron. 2016, 62, 226-234. [CrossRef]

17. Lee, Y.-H.; Kim, G.; Park, S.-R.; Lee, Y.-T.; Kim, N. An efficient emergency broadcasting signal multiplexing method for supporting the legacy T-DMB receivers in break-in system. IEEE Trans. Consum. Electron. 2011, 57, 1550-1555. [CrossRef]

18. Baek, M.-S.; Lee, Y.-H.; Kim, G.; Park, S.-R.; Lee, Y.-T. Development of T-DMB emergency broadcasting system and trial service with the legacy receivers. IEEE Trans. Consum. Electron. 2013, 59, 38-44. [CrossRef]

19. Choi, S.-J.; Kwon, D.-B.; Kim, J.-Y.; Oh, K.-S.; Chang, T.-U.; Hahm, Y.-K. Design of T-DMB automatic emergency alert service standard: Part 1 requirements analysis. J. Broadcast Eng. 2007, 12, 230-241. [CrossRef]

20. ATSC Standard: ATSC 3.0 System (A/300). September 2019.

21. ATSC Standard: System Discovery and Signaling (A/321). March 2016.

22. ATSC Standard: Physical Layer Protocol (A/322). December 2018.

23. ATSC Standard: Link-Layer Protocol (A/330). May 2019.

24. ATSC Standard: Signaling, Delivery, Synchronization, and Error Protection (A/331). June 2019. 
25. Gomez-Barquero, D.; Caldwell, M.W. Broadcast television spectrum incentive auctions in the U.S.: Trends, challenges, and opportunities. IEEE Commun. Mag. 2015, 53, 50-56. [CrossRef]

26. Kim, H.; Kim, J.; Park, S.I.; Lee, J.-Y.; Hur, N.; Simon, M.; Aitken, M.; Gage, K. An improved decoding scheme for emergency alert wake-up bits in ATSC 3.0. IEEE Trans. Broadcast. 2020, 66, 1-8. [CrossRef]

27. Kim, H.; Kim, J.; Park, S.I.; Lee, J.-Y.; Hur, N.; Simon, M.; Aitken, M.; Gage, K. A new decoding scheme of emergency alert wake-up bits in ATSC 3.0. In Proceedings of the IEEE International Symposium on Broadband Multimedia Systems and Broadcasting, Jeju, Korea, 5-7 June 2019.

28. 3GPP TS 24.502 V17.1.0: Access to the 3GPP 5G Core Network (5GCN) via Non-3GPP Access Networks (N3AN). Stage 3, Release 17. December 2020.

29. 3GPP TS 29.500 V16.5.0: 5G System; Technical Realization of Service Based Architecture. Stage 3, Release 16. September 2020.

30. Zhang, L.; Li, W.; Wu, Y.; Prasad, A.; Park, S.-I.; Hur, N. Using non-orthogonal multiplexing for enhancing unicast-broadcast transmission capacity in 5G. In Proceedings of the 2nd 5G World Forum, Dresden, Germany, 30 September-2 October 2019; pp. 214-219.

31. Simon, M.; Kofi, E.; Libin, L.; Aitken, M. ATSC 3.0 broadcast 5 G unicast heterogeneous network converged services starting release 16. IEEE Trans. Broadcast. 2020, 66, 449-458. [CrossRef]

32. Ghosh, A.; Maeder, A.; Baker, M.; Chandramouli, D. 5G evolution: A view on 5G cellular technology beyond $3 \mathrm{GPP}$ release 15. IEEE Access 2019, 7, 127639-127651. [CrossRef]

33. 3GPP TS 38.331 V15.8.0: Radio Resource Control (RRC) protocol specification. Release 15. December 2019.

34. 3GPP TS 29.518 V16.2.0: Access and Mobility Management Services. Stage 3, Release 16. December 2019.

35. Hikmaturokhman, A.; Suryanegara, M.; Ramli, K. A comparative analysis of 5G channel model with varied frequency: A case study in Jakarta. In Proceedings of the International Conference on Smart Computing \& Communications, Sarawak, Malaysia, 28-30 June 2019.

36. Digital Video Broadcasting (DVB): Implementation Guidelines for a Second Generation Digital Terrestrial Television Broadcasting System (DVB-T2), ETSI EN 102831 v1.2.1 2012-08.

37. Goldsmith, A. Wireless Communications; Cambridge University Press: Cambridge, UK, 2005. 